\title{
Interacting Induced Dark Energy Model
}

\author{
Amir F. Bahrehbakhsh* \\ Department of Physics, Faculty of Science, Payam-e-Noor University, Iran \\ Department of Physics and Astronomy, University of California, Irvine, CA 92697, USA
}

June 25, 2017

\begin{abstract}
Following the idea of the induced matter theory, for a non-vacuum five-dimensional version of general relativity, we propose a model in which the induced terms emerging from the extra dimension in our four-dimensional space-time, supposed to be as dark energy. Then we investigate the FLRW type cosmological equations and illustrate that when the scale factor of the fifth dimension has no dynamics, in early time the universe expands with deceleration and then in late time, expands with acceleration. In this case, the state parameter of the effective dark energy has a range of $-1<\bar{w}_{X}<0$ and it has the value $-1 / 2$ for present time. The results for current acceleration impose that $\Omega_{X \circ}>2 \Omega_{M \circ}$ which is in agreement with the measurements. We show that the effective energy density of dark energy have been having the same order of magnitude of the effective energy density of matter from the early time in the decelerating epoch of the universe expansion until now. The model avoids the cosmological coincidence problem.
\end{abstract}

Keywords: Extra Dimensions; Induced-Matter Theory; FLRW Cosmology; Dark Energy; Cosmological Coincidence Problem.

\section{Introduction}

According to the observations of distant type-Ia supernovae which indicate that the expansion of the universe is presently accelerating [1]- [5], the universe should mainly be filled with what is usually called dark energy [6], [7]. Therefore, a considerable amount of researches has been performed to explain this acceleration which, in general, can be classified into two groups. The first group contains all modifications to the standard model of cosmology: cosmological constant $(\Lambda \mathrm{CDM})$, modified matter models such as the quintessence [8]- [11], the k-essence [12]- [15], Chaplygin gas [16] models and also the models based on the modified theories of gravity like $f(R)$ gravity [17], [18], $f(T)$ gravity [19], [20] and $f(R, T)$ gravity [21].

The second group includes all the models based on the alternative theories of gravity such as Brans-Dicke (BD) theories [22]- [26], Kaluza-Klein (KK) theories [27]- [29], induced matter (IM) theories [30]- [33], brane world scenarios [34]- [38], Über gravity [39] and some mixture of these alternative theories [40]- [43] in addition to many other ones [44], [45]. Hence, in the recent two decades, explaining the accelerated expansion of the universe through the fundamental theories has been a great challenge.

Since, the most successful unifying theories such as string theory and M-theory work in higher dimensional space-time [46], in this work similar to the idea of IM theory, we try to find a geometrical origin, based on the fifth dimension, for dark energy. The significant of the IM theories is that inducing $5 D$ field equations without matter sources leads to the $4 D$ field equations with matter sources. In

*email: amirfarshad@gmail.com 
another word, the matter sources of $4 D$ space-times can be viewed as a manifestation of extra dimensions [30]- [32].

But, here we consider a non-vacuum $5 D$ version of general relativity (GR) and propose a model in which, neither the baryonic nor dark matter, but just only dark energy is considered as an effect of existence of the fifth dimension. We employ a generalized $5 D$ Friedmann-Lemaitre-RobertsonWalker (FLRW) type metric for the model and investigate its cosmological implications. In section 2, we give a brief review of the $5 D$ version of GR and induce the non-vacuum $5 D$ field equations in a $4 D$ hypersurface and then collect the extra terms emerging from the fifth dimension as dark energy component of the induced energy-momentum tensor in addition to the common energy-momentum tensor of the matter (the baryonic and dark matter). In section 3, we derive the FLRW cosmological equations by considering a generalized FLRW metric in a $5 D$ space-time. Then, we obtain the total energy conservation equation and separate it into two interacting energy conservation equations one for all kind of the matter and the other one for dark energy. In the following, we investigate three simple and important cases. First, we assume the fifth-dimension momentum density, $Q$, equals to zero and then we suppose the pressure in fifth dimension, $p_{5}$, be zero. Finally, we assume the scale factor of the fifth dimension to be a constant. At the end, in the last section conclusion is presented.

\section{Induced Dark Energy Model}

Following the approach of IM theories, one can consider a $5 D$ version of GR with action,

$$
S=\int \sqrt{\left|{ }^{(5)} g\right|}\left(\frac{1}{16 \pi G}{ }^{(5)} R+L_{m}\right) d^{5} x
$$

where $c=1,{ }^{(5)} R$ is $5 D$ Ricci scalar, ${ }^{(5)} g$ is the determinant of $5 D$ metric $g_{A B}$ and $L_{m}$ represents the matter Lagrangian. Hence, the Einstein field equations in five dimension can be written as

$$
{ }^{(5)} G_{A B}=8 \pi G{ }^{(5)} T_{A B},
$$

where the capital Latin indices run from zero to four, ${ }^{(5)} G_{A B}$ is $5 D$ Einstein tensor and ${ }^{(5)} T_{A B}$ is $5 D$ energy-momentum tensor. As a reasonable assumption we consider that ${ }^{(5)} T_{\alpha \beta}$ (the Greek indices go from zero to three) represents the same baryonic and dark matter sources of a $4 D$ hypersurface, i.e. $T_{\alpha \beta}^{(M)}$. We also take the $5 D$ energy-momentum tensor in the form of

$$
{ }^{(5)} T_{A B}=\left(\begin{array}{ccccc}
\rho_{M} & 0 & 0 & 0 & Q \\
0 & p_{M} & 0 & 0 & 0 \\
0 & 0 & p_{M} & 0 & 0 \\
0 & 0 & 0 & p_{M} & 0 \\
Q & 0 & 0 & 0 & p_{5}
\end{array}\right),
$$

where $\rho_{M}$ and $p_{M}$ are energy density and pressure of the matter (baryonic and dark matter) and also $p_{5}$ and $Q$ respectively represent the pressure and momentum density in fifth dimension. For cosmological purposes we restricts our attention to the $5 D$ warped metrics of the form

$$
d S^{2}=g_{A B}\left(x^{C}\right) d x^{A} d x^{B}={ }^{(5)} g_{\mu \nu}\left(x^{C}\right) d x^{\mu} d x^{\nu}+g_{44}\left(x^{C}\right) d y^{2} \equiv{ }^{(5)} g_{\mu \nu}\left(x^{C}\right) d x^{\mu} d x^{\nu}+\epsilon b^{2}\left(x^{C}\right) d y^{2},
$$

in local coordinates $x^{A}=\left(x^{\mu}, y\right)$, where $y$ represents the fifth coordinate and $\epsilon^{2}=1$. By assuming the $5 D$ space-time is foliated by a family of hypersurfaces, $\Sigma$, which are defined by fixed values of $y$, then, one can obtain the intrinsic metric of any typical hypersurface, e.g. $\Sigma_{\circ}\left(y=y_{\circ}\right)$, by restricting the line element (4) to displacements confined to it. Therefore, the induced metric on the hypersurface $\Sigma_{\circ}$ becomes

$$
d s^{2}={ }^{(5)} g_{\mu \nu}\left(x^{\alpha}, y_{\circ}\right) d x^{\mu} d x^{\nu} \equiv g_{\mu \nu} d x^{\mu} d x^{\nu},
$$


thus the usual $4 D$ space-time metric, $g_{\mu \nu}$, can be recovered. Therefore after some manipulations, equation (2) on the hypersurface $\Sigma_{\circ}$ can be written as

$$
G_{\alpha \beta}=8 \pi G\left(T_{\alpha \beta}^{(M)}+T_{\alpha \beta}^{(X)}\right)
$$

where we consider $T_{\alpha \beta}^{(X)}$ as dark energy component of the energy-momentum tensor that analogous to the IM theory [42] and [43], defined by

$$
\begin{array}{r}
T_{\alpha \beta}^{(X)} \equiv T_{\alpha \beta}^{(\mathrm{IM})} \equiv \frac{1}{8 \pi G}\left\{\frac{b_{; \alpha \beta}}{b}-\frac{\square b}{b} g_{\alpha \beta}-\frac{\epsilon}{2 b^{2}}\left[\frac{b^{\prime}}{b} g_{\alpha \beta}^{\prime}-g_{\alpha \beta}^{\prime \prime}+g^{\mu \nu} g_{\alpha \mu}^{\prime} g_{\beta \nu}^{\prime}-\frac{1}{2} g^{\mu \nu} g_{\mu \nu}^{\prime} g_{\alpha \beta}^{\prime}\right.\right. \\
\left.\left.\quad-g_{\alpha \beta}\left(\frac{b^{\prime}}{b} g^{\mu \nu} g_{\mu \nu}^{\prime}-g^{\mu \nu} g_{\mu \nu}^{\prime \prime}-\frac{1}{4} g^{\mu \nu} g^{\rho \sigma} g_{\mu \nu}^{\prime} g_{\rho \sigma}^{\prime}-\frac{3}{4} g^{\prime \mu \nu} g_{\mu \nu}^{\prime}\right)\right]\right\},
\end{array}
$$

in which the prime denotes derivative with respect to the fifth coordinate.

In the following section, we consider a generalized FLRW metric in a $5 D$ universe and investigate its cosmological properties.

\section{Generalized FLRW Cosmology}

For a $5 D$ universe with an extra space-like dimension in addition to the three usual spatially homogenous and isotropic ones, metric (4), as a generalized FLRW solution, can be written as

$$
d S^{2}=-d t^{2}+\tilde{a}^{2}(t, \tilde{y})\left[\frac{d r^{2}}{1-k r^{2}}+r^{2}\left(d \theta^{2}+\sin ^{2} \theta d \varphi^{2}\right)\right]+\tilde{b}^{2}(t, \tilde{y}) d \tilde{y}^{2} .
$$

Generally, the scale factors $\tilde{a}$ and $\tilde{b}$ should be functions of cosmic time and extra dimension coordinate. However, for physical plausibility and simplicity, we assume that they are separable functions of time and extra coordinate. Besides, the functionality of the scale factor $\tilde{b}$ on $\tilde{y}$ could be eliminated by transforming to a new extra coordinate $y$, hence metric (8) can be rewritten as

$$
d S^{2}=-d t^{2}+a^{2}(t) l^{2}(y)\left[\frac{d r^{2}}{1-k r^{2}}+r^{2}\left(d \theta^{2}+\sin ^{2} \theta d \varphi^{2}\right)\right]+b^{2}(t) d y^{2} .
$$

By considering metric (9) and assuming $H \equiv \dot{a} / a, B \equiv \dot{b} / b$ and $L \equiv l^{\prime} / l$, the Einstein equations (2) reduce as follows. The time component, $A=0=B$, provides

$$
H^{2}=\frac{8 \pi G}{3} \rho_{M}-H B+\frac{1}{b^{2}}\left(L^{\prime}+2 L^{2}\right)-\frac{k}{a^{2} l^{2}} \equiv \frac{8 \pi G}{3} \tilde{\rho}-\frac{k}{a^{2} l^{2}},
$$

the spatial ones, $A=B=1,2$ or 3 , give

$$
\frac{\ddot{a}}{a}=-4 \pi G p_{M}-\frac{1}{2} H^{2}-H B-\frac{1}{2}\left(\dot{B}+B^{2}\right)-\frac{1}{2 b^{2}}\left(2 L^{\prime}+3 L^{2}\right)-\frac{k}{2 a^{2} l^{2}} \equiv-\frac{4 \pi G}{3}(\tilde{\rho}+3 \tilde{p}),
$$

the $A=4=B$ component yields

$$
\frac{\ddot{a}}{a}=\frac{8 \pi G}{3} p_{5}-H^{2}+\frac{1}{b^{2}} L^{2}-\frac{k}{a^{2} l^{2}}
$$

and finally $A=0$ and $B=4$ (and also $A=4$ and $B=0$ ) component results

$$
L(H-B)=\frac{8 \pi G}{3} Q .
$$


In equations (10) and (11) we have defined $\tilde{\rho} \equiv \rho_{M}+\rho_{X}$ and $\tilde{p} \equiv p_{M}+p_{X}$ in which the energy density and pressure of dark energy has specified by employing relation (6), as

$$
\rho_{X} \equiv T_{\circ \circ}^{(X)}=\frac{3}{8 \pi G}\left[\frac{1}{b^{2}}\left(L^{\prime}+2 L^{2}\right)-H B\right]
$$

and

$$
p_{X} \equiv-T_{i i}^{(X)}=\frac{1}{8 \pi G}\left[\dot{B}+B^{2}+2 H B-\frac{1}{b^{2}}\left(2 L^{\prime}+3 L^{2}\right)\right] .
$$

By substituting equation (10) in (12) and assuming $p_{M}=0$ for current important components of the matter (baryonic and dark matter), one has

$$
b^{2}\left[\dot{H}+H^{2}-H B+\frac{8 \pi G}{3}\left(\rho_{M}-p_{5}\right)\right]=-\left(L^{\prime}+L^{2}\right)=-\lambda^{2},
$$

where $\lambda$ is a non-zero real constant and obtained from the fact that the left hand side of the equation is a function of cosmic time but the right hand side of the equation is a function of extra dimension coordinate, thus both sides of the equation must be equal to a constant. From the equation (16) one can get

$$
l(y)=l_{\circ} e^{ \pm \lambda\left(y-y_{\circ}\right)},
$$

with $L= \pm \lambda$ and $L^{\prime}=0$. Hence, the equations (10)-(15) on the hypersurface $\Sigma_{\circ}\left(y=y_{\circ}\right)$ can be rewritten as

$$
\begin{gathered}
H^{2}=\frac{8 \pi G}{3} \rho_{M}-H B+\frac{2 \lambda^{2}}{b^{2}}-\frac{k}{a^{2} l_{\circ}^{2}} \equiv \frac{8 \pi G}{3} \tilde{\rho}-\frac{k}{a^{2} l_{\circ}^{2}}, \\
\frac{\ddot{a}}{a}=\frac{8 \pi G}{3} p_{5}-H^{2}+\frac{\lambda^{2}}{b^{2}}-\frac{k}{a^{2} l_{\circ}^{2}} \equiv-\frac{4 \pi G}{3}(\tilde{\rho}+3 \tilde{p}), \\
H-B=\frac{8 \pi G}{3 \lambda} Q, \\
\rho_{X}=\frac{3}{8 \pi G}\left(\frac{2 \lambda^{2}}{b^{2}}-H B\right)
\end{gathered}
$$

and

$$
p_{X}=\frac{1}{8 \pi G}\left(\dot{B}+B^{2}+2 H B-\frac{3 \lambda^{2}}{b^{2}}\right),
$$

in which

$$
p_{5}=\frac{1}{2}\left(\rho_{M}+\rho_{X}-3 p_{X}\right)-\frac{3 \lambda^{2}}{8 \pi G b^{2}} .
$$

On the other hand, to obtain energy conservation equation, one can take the time derivative of the equation (10) and substitute the equation (11) into it and get

$$
\dot{\tilde{\rho}}+3 H(\tilde{\rho}+\tilde{p})=0 .
$$

As the dark energy density is of the same order as the dark matter energy density in the present universe, one can imagine that there is some connection between dark energy and dark matter and 
expect their conservation equations may not to be independent. Hence, we assume equation (24) can plausibly separate into two distinguished equations for $\rho_{X}$ and $\rho_{M}$ as

$$
\dot{\rho}_{X}+3 H\left(\rho_{X}+p_{X}\right)=f(t)
$$

and

$$
\dot{\rho}_{M}+3 H\left(\rho_{M}+p_{M}\right)=-f(t)
$$

where $f(t)$ is assumed to be the interacting term between dark energy and dark matter.

Now, substituting relations (21) and (22) into the equation (25), gets

$$
b^{2}\left[\dot{H}+H^{2}-H B+\frac{8 \pi G}{3} \frac{f(t)}{B}\right]=\frac{\lambda^{2}}{B}(3 H-4 B),
$$

which comparing it with the equation (16) results

$$
f(t)=B\left(\rho_{M}-p_{5}\right)+\frac{3 \lambda Q}{b^{2}} .
$$

In the following, we investigate three cases. First, we assume the fifth-dimension momentum density be zero, i.e. $Q=0$. Then we suppose the pressure in fifth dimension, $p_{5}$, equals to zero. Finally, we assume the scale factor of the fifth dimension to be a constant.

\subsection{The Model with Zero Fifth-Dimension Momentum Density; $Q=0$}

By considering $Q=0$, from the equation (20) one has $B=H$ with $b=\left(a_{\circ} / b_{\circ}\right) a$. Hence the equations (18) and (19) and the interacting term between matter and dark energy become

$$
\begin{aligned}
& H^{2}=\frac{4 \pi G}{3} \rho_{M}+\frac{a_{\circ}^{2} \lambda^{2}}{b_{\circ}^{2} a^{2}}-\frac{k}{2 l_{\circ}^{2} a^{2}}, \\
& \frac{\ddot{a}}{a}=-\frac{4 \pi G}{3}\left(\rho_{M}-2 p_{5}\right)-\frac{k}{2 l_{\circ}^{2} a^{2}}
\end{aligned}
$$

and

$$
f(t)=H\left(\rho_{M}-p_{5}\right) .
$$

In the case $f(t)=0$, one has $p_{5}=\rho_{M}$ and from the equations (26) gets

$$
\rho_{M}=\rho_{M \circ}\left(\frac{a_{\circ}}{a}\right)^{3}
$$

and hence equation (30) becomes

$$
\frac{\ddot{a}}{a}=\frac{4 \pi G \rho_{M \circ}}{3}\left(\frac{a_{\circ}}{a}\right)^{3}-\frac{k}{2 l_{\circ}^{2} a^{2}} .
$$

For spatially flat or open universe $(k=0$ and -1$)$, equation (33) illustrates eternal accelerated expansion, but for a closed one, it suggests that the expansion of the universe first accelerates and then decelerates. Therefore, the non-interacting case for all kind of the geometry has results contrary to the observations and hence it is not a suitable case.

When $f(t) \neq 0$, from the acceleration equation (30), it is clear that the first term containing energy density of matter, $\rho_{M}$, only contributes in deceleration of the universe expansion. However, the second and third terms are capable to contributes in the acceleration but, multiplying the equation (29) by $a^{2}$, shows that these terms also can not increase the velocity of the universe expansion, i.e. $\dot{a}$. Hence the model, when the fifth-dimension momentum density is zero, is not self consistent to having the accelerating solutions. Therefore, in the following as the second case we assume the fifth-dimension pressure to be zero. 


\subsection{The Model with Zero Fifth-Dimension Pressure; $p_{5}=0$}

Taking $p_{5}=0$ with a mathematically simplification and physically plausible assumption as $B=$ $n H$ gives

$$
Q=\frac{3 \lambda}{8 \pi G}(1-n) H
$$

and

$$
f(t)=\left(n \rho_{M}+\frac{9 \lambda^{2}}{8 \pi G} \frac{1-n}{a^{2 n}}\right) H,
$$

which substituting the second one in the equation (26) leads

$$
\rho_{M}=\rho_{M \circ}\left(\frac{a_{\circ}}{a}\right)^{n+3}+\beta \frac{n-1}{3-n} a^{-2 n} .
$$

Then, equations (18) and (19) become

$$
H^{2}=\frac{8 \pi G \rho_{M \circ}}{3(n+1)}\left(\frac{a_{\circ}}{a}\right)^{n+3}+\frac{n+3}{(n+1)(3-n)} \frac{\lambda^{2}}{a^{2 n}}-\frac{k}{(n+1) l_{\circ}^{2} a^{2}}
$$

and

$$
\frac{\ddot{a}}{a}=-\frac{8 \pi G \rho_{M \circ}}{3(n+1)}\left(\frac{a_{\circ}}{a}\right)^{n+3}+\frac{n(1-n)}{(n+1)(3-n)} \frac{\lambda^{2}}{a^{2 n}}-\frac{n k}{(n+1) l_{\circ}^{2} a^{2}} .
$$

The consistency condition between the equations (35) yields to $n=1$, which gives the same unsuit-

able results of the previous case with $Q=0$. Therefore, as the last assumption we consider that the scale factor of the fifth dimension does not have any dynamics.

\subsection{The Model with Static Scale Factor of the Fifth-Dimension; $b=$ constant}

Assuming $b=b_{\circ}=c t e$, gets $B=0$ and from the equations (18)-(22) one has

$$
\begin{aligned}
& H^{2}=\frac{8 \pi G}{3}\left(\rho_{M}+\rho_{X}\right)-\frac{k}{l_{\circ}^{2} a^{2}}, \\
& \frac{\ddot{a}}{a}=-\frac{4 \pi G}{3}\left(\rho_{M}+\rho_{X}+3 p_{X}\right)
\end{aligned}
$$

and

$$
Q=\frac{3 \lambda}{8 \pi G} H
$$

in which

$$
\rho_{X}=\frac{3 \lambda^{2}}{4 \pi G b_{\circ}^{2}}
$$

and

$$
p_{X}=-\frac{3 \lambda^{2}}{8 \pi G b_{\circ}^{2}} .
$$

Also, from the equations (28), (41) and (42) one gets

$$
f(t)=\frac{9 \lambda^{2}}{4 \pi G b_{\circ}^{2}} H=\frac{3}{2} \rho_{X} H,
$$




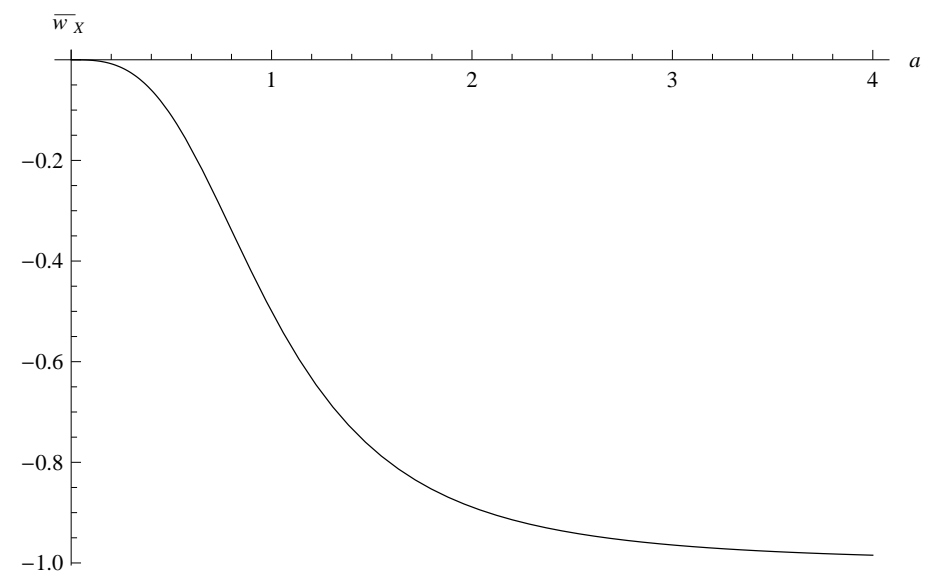

Figure 1: Changes of state parameter of effective dark energy with evolution of the scale factor of the ordinary spatial dimensions by setting $a_{\circ}=1$.

thereupon, substituting the relation (44) in the equation (26) gives

$$
\rho_{M}=\rho_{M \circ}\left(\frac{a_{\circ}}{a}\right)^{3}+\frac{3 \lambda^{2}}{8 \pi G b_{\circ}^{2}}\left[\left(\frac{a_{\circ}}{a}\right)^{3}-1\right] .
$$

To have the common form of the evolution of energy density of matter, we define the effective energy densities of matter and dark energy as follows

$$
\bar{\rho}_{M} \equiv \rho_{M \circ}\left(\frac{a_{\circ}}{a}\right)^{3}
$$

and

$$
\bar{\rho}_{X} \equiv \frac{3 \lambda^{2}}{8 \pi G b_{\circ}^{2}}\left[\left(\frac{a_{\circ}}{a}\right)^{3}+1\right]
$$

Hence, the equations (39) and (40) can be rewritten as

$$
H^{2}=\frac{8 \pi G}{3}\left(\bar{\rho}_{M}+\bar{\rho}_{X}\right)-\frac{k}{l_{\circ}^{2} a^{2}} \equiv \frac{8 \pi G}{3} \rho_{M \circ}\left(\frac{a_{\circ}}{a}\right)^{3}+\frac{\lambda^{2}}{b_{\circ}^{2}}\left[1+\left(\frac{a_{\circ}}{a}\right)^{3}\right]-\frac{k}{l_{\circ}^{2} a^{2}}
$$

and

$$
\frac{\ddot{a}}{a}=-\frac{4 \pi G}{3}\left(\bar{\rho}_{M}+\bar{\rho}_{X}+3 \bar{p}_{X}\right) \equiv-\frac{4 \pi G}{3} \rho_{M \circ}\left(\frac{a_{\circ}}{a}\right)^{3}+\frac{\lambda^{2}}{b_{\circ}^{2}}\left[1-\frac{1}{2}\left(\frac{a_{\circ}}{a}\right)^{3}\right]
$$

in which

$$
\bar{p}_{X}=p_{X}=-\frac{3 \lambda^{2}}{8 \pi G b_{\circ}^{2}} .
$$

Then, the state parameter of effective dark energy becomes

$$
\bar{w}_{X}=\frac{\bar{p}_{X}}{\bar{\rho}_{X}}=-\frac{a^{3}}{a^{3}+a_{\circ}^{3}},
$$

which has a range of $-1<\bar{w}_{X}<0$. Fig. 1 shows the changes of state parameter of effective dark energy. 
Since the measurements of anisotropies in the cosmic microwave background indicates that the universe must be very close to spatially flat one [47]-[49], then we consider $k=0$. Equation (49) illustrates that the universe expansion, in the early epoch, decelerates and then in the late epoch, accelerates. Present acceleration imposes $\frac{\lambda^{2}}{b_{\circ}^{2}}>\frac{8 \pi G}{3} \rho_{M \circ}$, this yields to $\Omega_{X \circ}>2 \Omega_{M \circ}$, or, $\Omega_{X \circ}>2 / 3$ and $\Omega_{M \circ}<1 / 3$, which is in agreement with the measurements, i.e. $\Omega_{X \circ} \approx 0.7$ and $\Omega_{M \circ} \approx 0.3$.

Also, one gets

$$
\frac{\bar{\rho}_{X}}{\bar{\rho}_{M}}=\frac{3 \lambda^{2}}{8 \pi G b_{\circ}^{2} \rho_{M \circ}} \frac{1}{1+\bar{w}_{X}}>\frac{1}{1+\bar{w}_{X}}=\left\{\begin{array}{lll}
1 & \bar{w}_{X} \rightarrow 0 & (a \rightarrow 0) \\
2 & \bar{w}_{X}=-1 / 2 & \left(a=a_{\circ}\right) \\
+\infty & \bar{w}_{X} \rightarrow-1 & (a \rightarrow \infty),
\end{array}\right.
$$

which shows that the effective energy density of dark energy dominates all the time of the universe expansion with the same order of magnitude of energy density of matter in the past and present time. Hence, the cosmological coincidence problem can be solved with this model. Note, this domination of dark energy does not mean eternally accelerating expansion in this model, the term, $-\frac{\lambda^{2}}{2 b_{\circ}^{2}}\left(\frac{a_{\circ}}{a}\right)^{3}$, actually contributes in deceleration, so at early time beside the energy density of matter, the effective energy density of dark energy also contributes in deceleration.

At the end, we should mention that the results of GW170817 apply only to large extra dimensions because, gravitational waves detected from the neutron star collision have wavelengths of thousands of kilometers and would not affect tiny extra dimensions. Hence, this imposes that $b_{\circ}$ must be very small.

\section{Conclusions}

The observations illustrate that the universe is presently in an accelerated expanding phase. Hence, the main content of the universe should be consisted of what commonly called dark energy. However, an enormous amount of work has been performed to explain this acceleration, but the origin and the nature of dark energy is unknown yet.

In this work, following the approach of the induced matter theory, we have investigated the cosmological implications of a non-vacuum five-dimensional version of general relativity in order to explain both deceleration and acceleration eras of the universe expansion and solve the cosmological coincidence problem. In this respect, in general we have considered a five dimensional energymomentum tensor contains the elements, pressure and momentum density in fifth dimension, i.e., $p_{5}$ and $Q$, in addition to the energy density and the pressure of the matter (baryonic and dark matter) ones. Then, on a $4 D$ hypersurface, we have classified the energy-momentum tensor into two parts. One part represents the baryonic and dark matter together and the other one which contains every extra terms emerging from the fifth dimension, have been considered as the energy-momentum tensor of dark energy. Then, by considering a generalized FLRW metric in a $5 D$ space-time, we have derived the FLRW cosmological equations on the $4 D$ hypersurface and separated the total energy conservation equation into the two equation, one for the matter and the other one for dark energy with interacting term between them. Afterwards, we have investigated three cases.

In the first one, we assumed the fifth-dimension momentum density be zero, $Q=0$. In this case, for non-interacting situation for all kind of the geometry the results are contrary to the observations and also for interacting one the equations are not self consistent. For the second case, we supposed the pressure in the fifth dimension equals to zero, i.e. $p_{5}=0$. The consistency condition between the equations yields to the same unsuitable results of the first case with $Q=0$.

Finally, in the last case, we considered a tiny constant scale factor of the fifth dimension, i.e. $b=$ cte. In this situation by defining the effective energy densities of matter and dark energy we have illustrated that in early time, the universe expands with deceleration and then in the late time, expands with acceleration. The present acceleration, for this model, yields to $\Omega_{X \circ}>2 \Omega_{M \circ}$ which is in agreement with the measurements. The state parameter of the effective dark energy has the range 
$-1<\bar{w}_{X}<0$ and it is equal to $-1 / 2$ at the present time. We have also shown that the effective energy density of dark energy have has the same order of magnitude of the effective energy density of matter since the early time until now. Therefore, the model avoids the cosmological coincidence problem.

\section{Acknowledgements}

I would like to thank Department of Physics and Astronomy, University of California, Irvine, for the visit opportunity and their accommodations. Also, especial thanks to Tim Tait and Arvind Rajaraman for reading this article and useful comments.

\section{References}

[1] S. Perlmutter et al. [Supernova Cosmology Project Collaboration], "Discovery of a supernova explosion at half the age of the Universe and its cosmological implications," Nature 391, 51 (1998) doi:10.1038/34124 [astro-ph/9712212].

[2] A. G. Riess et al. [Supernova Search Team], "Observational evidence from supernovae for an accelerating universe and a cosmological constant," Astron. J. 116, 1009 (1998) doi:10.1086/300499 [astro-ph/9805201].

[3] S. Perlmutter et al. [Supernova Cosmology Project Collaboration], "Measurements of Omega and Lambda from 42 high redshift supernovae," Astrophys. J. 517, 565 (1999) doi:10.1086/307221 [astro-ph/9812133].

[4] P. de Bernardis et al., "Multiple peaks in the angular power spectrum of the cosmic microwave background: Significance and consequences for cosmology," Astrophys. J. 564, 559 (2002) doi:10.1086/324298 [astro-ph/0105296].

[5] S. M. Carroll, "Why is the universe accelerating?," eConf C 0307282, TTH09 (2003) [AIP Conf. Proc. 743, 16 (2005)] doi:10.1063/1.1848314 [astro-ph/0310342].

[6] V. Sahni, "Dark matter and dark energy," Lect. Notes Phys. 653, 141 (2004) doi:10.1007/b99562 [astro-ph/0403324].

[7] M. Kamionkowski, "Dark Matter and Dark Energy," [arXiv:0706.2986 [astro-ph]].

[8] I. Zlatev, L. M. Wang and P. J. Steinhardt, "Quintessence, cosmic coincidence, and the cosmological constant," Phys. Rev. Lett. 82, 896 (1999) doi:10.1103/PhysRevLett.82.896 [astro-ph/9807002].

[9] A. Albrecht and C. Skordis, "Phenomenology of a realistic accelerating universe using only Planck scale physics," Phys. Rev. Lett. 84, 2076 (2000) doi:10.1103/PhysRevLett.84.2076 [astro$\mathrm{ph} / 9908085]$.

[10] M. C. Bento, O. Bertolami and N. M. C. Santos, "A Two field quintessence model," Phys. Rev. D 65, 067301 (2002) doi:10.1103/PhysRevD.65.067301 [astro-ph/0106405].

[11] D. Blais and D. Polarski, "Transient accelerated expansion and double quintessence," Phys. Rev. D 70, 084008 (2004) doi:10.1103/PhysRevD.70.084008 [astro-ph/0404043].

[12] C. Armendariz-Picon, V. F. Mukhanov and P. J. Steinhardt, "Essentials of k essence," Phys. Rev. D 63, 103510 (2001) doi:10.1103/PhysRevD.63.103510 [astro-ph/0006373].

[13] C. Armendariz-Picon, V. F. Mukhanov and P. J. Steinhardt, "A Dynamical solution to the problem of a small cosmological constant and late time cosmic acceleration," Phys. Rev. Lett. 85, 4438 (2000) doi:10.1103/PhysRevLett.85.4438 [astro-ph/0004134]. 
[14] A. Y. Kamenshchik, U. Moschella and V. Pasquier, "An Alternative to quintessence," Phys. Lett. B 511, 265 (2001) doi:10.1016/S0370-2693(01)00571-8 [gr-qc/0103004].

[15] R. J. Scherrer, "Purely kinetic k-essence as unified dark matter," Phys. Rev. Lett. 93, 011301 (2004) doi:10.1103/PhysRevLett.93.011301 [astro-ph/0402316].

[16] M. C. Bento, O. Bertolami and A. A. Sen, "Generalized Chaplygin gas, accelerated expansion and dark energy matter unification," Phys. Rev. D 66, 043507 (2002) doi:10.1103/PhysRevD.66.043507 [gr-qc/0202064].

[17] S. Nojiri and S. D. Odintsov, "Introduction to modified gravity and gravitational alternative for dark energy," eConf C 0602061, 06 (2006) [Int. J. Geom. Meth. Mod. Phys. 4, 115 (2007)] doi:10.1142/S0219887807001928 [hep-th/0601213].

[18] H. J. Schmidt, "Fourth order gravity: Equations, history, and applications to cosmology," eConf C 0602061, 12 (2006) [Int. J. Geom. Meth. Mod. Phys. 4, 209 (2007)] doi:10.1142/S0219887807001977 [gr-qc/0602017].

[19] B. Li, T. P. Sotiriou and J. D. Barrow, " $f(T)$ gravity and local Lorentz invariance," Phys. Rev. D 83, 064035 (2011) doi:10.1103/PhysRevD.83.064035 [arXiv:1010.1041 [gr-qc]].

[20] R. Myrzakulov, "Accelerating universe from F(T) gravity," Eur. Phys. J. C 71, 1752 (2011) doi:10.1140/epjc/s10052-011-1752-9 [arXiv:1006.1120 [gr-qc]].

[21] T. Harko, F. S. N. Lobo, S. Nojiri and S. D. Odintsov, " $f(R, T)$ gravity," Phys. Rev. D 84, 024020 (2011) doi:10.1103/PhysRevD.84.024020 [arXiv:1104.2669 [gr-qc]].

[22] P. Jordan, "Projective Relativity," Friedr. View. Sohn, Braunschweig, (1955).

[23] C. Brans and R. H. Dicke, "Mach's principle and a relativistic theory of gravitation," Phys. Rev. 124, 925 (1961).

[24] R. H. Dicke, "Mach's principle and a relativistic theory of gravitation. ii," Phys. Rev. 125, 2194 (1962).

[25] B. Bertotti, L. Iess and P. Tortora, "A test of general relativity using radio links with the Cassini spacecraft," Nature 425, 374 (2003) doi:10.1038/nature01997.

[26] Y. Fujii and K. Maeda, "The Scalar-Tensor Theory of Gravitation," Cambridge University Press, Cambridge (2004) doi:10.1017/CBO9780511535093.

[27] T. Kaluza, "On the Problem of Unity in Physics," Sitz. Preuss. Akad. Wiss. 33, 966 (1921).

[28] O. Klein, "Quantum Theory and Five-Dimensional Theory of Relativity," Z. Phys. 37, 895 (1926) doi: $10.1007 / \mathrm{BF} 01397481$.

[29] J. M. Overduin and P. S. Wesson, "Kaluza-Klein gravity," Phys. Rept. 283, 303 (1997) doi:10.1016/S0370-1573(96)00046-4 [gr-qc/9805018].

[30] P. S. Wesson, "Space-Time-Matter, Modern Kaluza-Klein Theory," World Scientific, Singapore (1999) doi:10.1142/3889.

[31] P. S. Wesson, "Five-Dimensional Physics," World Scientific, Singapore (2006) doi:10.1142/6029.

[32] P. S. Wesson, "The status of modern five-dimensional gravity (A short review: Why physics needs the fifth dimension)," Int. J. Mod. Phys. D 24, no. 01, 1530001 (2014) doi:10.1142/S0218271815300013 [arXiv:1412.6136 [gr-qc]]. 
[33] A. F. Bahrehbakhsh, "FLRW Cosmology of Induced Dark Energy Model and Open Universe," Can. J. Phys. 95, 1215 (2017) doi:10.1139/cjp-2016-0827 [arXiv:1705.06506 [gr-qc]].

[34] L. Randall and R. Sundrum, "A Large mass hierarchy from a small extra dimension," Phys. Rev. Lett. 83, 3370 (1999) doi:10.1103/PhysRevLett.83.3370 [hep-ph/9905221].

[35] K. Ghoroku, M. Tachibana and N. Uekusa, Phys. Rev. D 68, 125002 (2003) doi:10.1103/PhysRevD.68.125002 [hep-th/0304051].

[36] I. H. Brevik and A. Hallanger, "Randall-Sundrum model in the presence of a brane bulk viscosity," Phys. Rev. D 69, 024009 (2004) doi:10.1103/PhysRevD.69.024009 [gr-qc/0308058].

[37] M. D. Maia, E. M. Monte and J. M. F. Maia, "The Accelerating universe in brane world cosmology," Phys. Lett. B 585, 11 (2004) doi:10.1016/j.physletb.2003.12.079 [astro-ph/0208223].

[38] R. Maartens and K. Koyama, "Brane-World Gravity," Living Rev. Rel. 13, 5 (2010) doi:10.12942/lrr-2010-5 [arXiv:1004.3962 [hep-th]].

[39] N. Khosravi, "Über-Gravity and the Cosmological Constant Problem," arXiv:1703.02052 [gr-qc].

[40] J. P. de Leon, "Late time cosmic acceleration from vacuum Brans-Dicke theory in 5D," Class. Quant. Grav. 27, 095002 (2010) doi:10.1088/0264-9381/27/9/095002 [arXiv:0912.1026 [gr-qc]].

[41] J. Ponce de Leon, "Brans-Dicke Cosmology in 4D from scalar-vacuum in 5D" JCAP 1003, 030 (2010) doi:10.1088/1475-7516/2010/03/030 [arXiv:1001.1961 [gr-qc]].

[42] A. F. Bahrehbakhsh, M. Farhoudi and H. Shojaie, "FRW Cosmology From Five Dimensional Vacuum Brans-Dicke Theory," Gen. Rel. Grav. 43, 847 (2011) doi:10.1007/s10714-010-1101-6 [arXiv:1005.2501 [gr-qc]].

[43] A. F. Bahrehbakhsh, M. Farhoudi and H. Vakili, "Dark Energy From Fifth Dimensional Brans-Dicke Theory," Int. J. Mod. Phys. D 22, 1350070 (2013) doi:10.1142/S0218271813500703 [arXiv:1306.1943 [gr-qc]].

[44] T. Clifton, P. G. Ferreira, A. Padilla and C. Skordis, "Modified Gravity and Cosmology," Phys. Rept. 513, 1 (2012) doi:10.1016/j.physrep.2012.01.001 [arXiv:1106.2476 [astro-ph.CO]].

[45] J. Yoo and Y. Watanabe, "Theoretical Models of Dark Energy," Int. J. Mod. Phys. D 21, 1230002 (2012) [arXiv:1212.4726 [astro-ph.CO]].

[46] M. Pavsic, "The Landscape of theoretical physics: A Global view. From point particles to the brane world and beyond, in search of a unifying principle," Fundam. Theor. Phys. 119 (2001) [gr-qc/0610061].

[47] N. A. Bahcall, J. P. Ostriker, S. Perlmutter and P. J. Steinhardt, "The Cosmic triangle: Assessing the state of the universe," Science 284, 1481 (1999) doi:10.1126/science.284.5419.1481 [astro$\mathrm{ph} / 9906463]$.

[48] P. de Bernardis et al. [Boomerang Collaboration], "A Flat universe from high resolution maps of the cosmic microwave background radiation," Nature 404, 955 (2000) doi:10.1038/35010035 [astro-ph/0004404].

[49] S. Hanany et al., "MAXIMA-1: A Measurement of the cosmic microwave background anisotropy on angular scales of 10 arcminutes to 5 degrees," Astrophys. J. 545, L5 (2000) doi:10.1086/317322 [astro-ph/0005123]. 